\title{
International experience with conversion from cyclosporine to tacrolimus for acute and chronic lung allograft rejection
}

Kambiz Sarahrudi, MD, ${ }^{\text {a }}$ Marc Estenne, MD, ${ }^{\mathrm{b}}$ Paul Corris, MD, ${ }^{\mathrm{c}}$ Jost Niedermayer, MD, ${ }^{\mathrm{d}}$ Christiane Knoop, MD, ${ }^{\mathrm{b}}$ Allan Glanville, MD, ${ }^{e}$ Cecilia Chaparro, MD, ${ }^{f}$ Geert Verleden, MD, ${ }^{g}$ Margaret W. Gerbase, MD, ${ }^{\mathrm{h}}$ Federico Venuta, MD, Heidi Böttcher, MD, John D. Aubert, MD, ${ }^{\text {, }}$ Bronwyn Levvey, MD, ${ }^{\prime}$ Hermann Reichenspurner, MD, ${ }^{m}$ Alexandra Auterith, ${ }^{n}$ and Walter Klepetko, MD

From the Department of Cardiothoracic Surgery, ${ }^{a}$ University of Vienna, Vienna, Austria; the Department of Pulmonology, Erasme University Hospital, Brussels, Belgium; the Department of Pulmonology, ${ }^{\mathrm{c}}$ Freeman Hospital, Newcastle, United Kingdom; the Department of Pulmonology, ${ }^{\mathrm{d}}$ University of Hannover, Hannover, Germany; the Department of Pulmonology, ${ }^{\mathrm{e}}$ St Vincent's Hospital, Sydney, Australia; the Department of Pulmonology, ${ }^{\mathrm{f}}$ University of Toronto, Toronto, Canada; the Department of Pulmonology, ${ }^{\mathrm{g}}$ University Hospital Gasthuisberg, Leuven, Belgium; the Department of Pulmonology, ${ }^{\text {h }}$ University of Geneva, Geneva, Switzerland; the Department of Thoracic Surgery, ${ }^{\mathrm{i}}$ University of Rome, Rome, Italy; the Department of Cardiovascular Surgery, ${ }^{j}$ University of Kiel, Kiel, Germany; the Department of Pulmonology, ${ }^{\mathrm{k}}$ University of Lausanne, Lausanne, Switzerland; the Department of Respiratory Medicine, ${ }^{1}$ The Alfred Hospital, Sydney, Australia; the Department of Cardiothoracic Surgery, ${ }^{\mathrm{m}}$ University of Munich, Munich, Germany; and the Department of Medical Statistics, ${ }^{\mathrm{n}}$ University of Vienna, Vienna, Austria.

Received for publication April 9, 2003; revisions requested Nov 3, 2003; accepted for publication Nov 10, 2003.

Address for reprints: Walter Klepetko, MD, Department of Cardiothoracic Surgery, University of Vienna, Austria, Währinger Gürtel 18-20, A-1090 Vienna, Austria (Email: walter.klepetko@akh-wien.ac.at).

J Thorac Cardiovasc Surg 2004;127:1126-32 $0022-5223 / \$ 30.00$

Copyright (C) 2004 by The American Association for Thoracic Surgery

doi:10.1016/j.jtcvs.2003.11.009
Objective: A retrospective study involving 13 institutions was performed to assess the efficacy of conversion from cyclosporine (INN: ciclosporin) to tacrolimus.

Methods: Data from 244 patients were analyzed. Indications for conversion were recurrent-ongoing rejection $(\mathrm{n}=110)$ and stage 1 to 3 bronchiolitis obliterans syndrome $(\mathrm{n}=134)$.

Results: The incidence of acute rejection decreased significantly within 3 months after versus before the switch from cyclosporine to tacrolimus $(P<.01)$. For patients with recurrent-ongoing rejection, the forced expiratory volume in 1 second decreased by $1.96 \%$ of predicted value per month $(P=.08$ vs zero slope) before and increased by $0.34 \%$ of predicted value per month $(P=.32$ vs zero slope $)$ after conversion $(P<.06)$. For patients with stage 1 to 3 bronchiolitis obliterans syndrome, a significant reduction of rejection episodes was observed $(P<.01)$. In single transplant recipients a decrease of the forced expiratory volume in 1 second averaged $2.25 \%$ of predicted value per month $(P<.01$ vs zero slope) before and $0.29 \%$ of predicted value per month after conversion. Corresponding values for bilateral transplant recipients were $3.7 \%$ of predicted value per month $(P<.01$ vs zero slope) and $0.9 \%$ of predicted value per month $(P=0.04$ vs zero slope), respectively. No significant difference in the incidence of infections within 3 months before and after conversion was observed.

Conclusions: Conversion from cyclosporine to tacrolimus after lung transplantation is associated with reversal of recurrent-ongoing rejection. Conversion for bronchiolitis obliterans syndrome allows short-term stabilization of lung function in most patients.

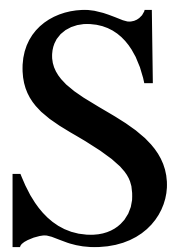

hort-term outcome after lung transplantation has improved significantly over the last decade, but long-term survival remains limited by the development of bronchiolitis obliterans syndrome (BOS), ${ }^{1-3}$ which is believed to be a manifestation of chronic allograft rejection and occurs in as much as $30 \%$ to $50 \%$ of patients at 2 years after transplantation. Clinical reports have demonstrated that acute rejection (AR), especially when histologically severe or refractory to treatment, is the primary risk factor for BOS. ${ }^{1-8}$ Tacrolimus, which is a more potent immunosuppressive agent than cyclosporine (INN: ciclosporin) in vitro, ${ }^{9}$ has been successfully used in the treatment of intractable acute and chronic rejection after kidney, ${ }^{10}$ liver, ${ }^{11}$ and heart ${ }^{12}$ transplantation. After these reports, several studies have assessed tacrolimus as rescue therapy for recurrent-ongoing acute and chronic rejection after lung transplantation. They suggested that conversion from cyclosporine to tacrolimus significantly reduces the incidence and severity of $\mathrm{AR}^{13-17}$ and might stabilize 
pulmonary function in patients with BOS. ${ }^{15,18-20}$ These results, however, are difficult to interpret because they come from single-center studies that included small numbers of patients (ie, between 10 and 15) and did not allow stratification according to the indication for conversion or type of transplant procedure. To overcome these limitations, we undertook this retrospective multicenter study to assess the effects of conversion from cyclosporine to tacrolimus on recurrent-ongoing acute rejection (RAR) and BOS in a larger cohort of lung transplant recipients.

\section{Methods \\ Patients}

Data from 13 European, Australian, and Canadian lung transplantation centers were collected by means of questionnaire. Each center was asked for clinical data of all patients ever converted from cyclosporine to tacrolimus for RAR, BOS, or both up to December 2000. Data included (1) patient's age and sex; (2) indication for transplantation, type of transplantation, and time from transplantation to conversion; (3) duration of follow-up and outcome; (4) immunosuppression at the time of conversion; (5) incidence of episodes of AR within 3 months before and 3 months after conversion; (6) values of forced expiratory volume in 1 second $\left(\mathrm{FEV}_{1}\right)$ at 3 and 6 months before conversion, at the time of conversion, and at 3, 6, and 12 months after conversion; and (7) incidence of infectious complications and values of serum creatinine within 3 months before and 3 months after conversion.

Patients were grouped according to the indication for conversion. Group 1 (RAR) included patients who were switched from cyclosporine to tacrolimus for RAR, which was defined as 2 or more episodes of rejection within 3 months before conversion, as diagnosed on the basis histology $\left(>\mathrm{A}_{1}\right)$ or clinical changes (eg, a decrease in lung function or a change in chest radiographic results that responded to augmented immunosuppression and was not explained by other causes), and patients who were switched for refractory (ongoing) $\mathrm{AR}$ (defined by $>\mathrm{A}_{1}$ histologic changes on consecutive sets of biopsies). Group 2 (BOS) included patients who had BOS of greater than stage 0 , as defined by the International Society for Heart and Lung Transplantation. ${ }^{21}$ This classification indicates that a patient with an $\mathrm{FEV}_{1}$ value of between $100 \%$ and $81 \%$ of the best postoperative value is in BOS stage 0 ; corresponding $\mathrm{FEV}_{1}$ values for BOS stages 1, 2, and 3 are $66 \%$ to $80 \%, 51 \%$ to $65 \%$, and $50 \%$ or less of baseline values, respectively. Conversion of cyclosporine to tacrolimus was also performed at some centers for patients who showed a decrease in $\mathrm{FEV}_{1}$ of less than $19 \%$ compared with the baseline value, a decrease in the midexpiratory flow rate, or both; these patients were categorized as being in pre-BOS, stage, and their data were not analyzed in this study.

Patients with a combination of RAR and BOS were allocated to the BOS group. For analysis of procedure-related results, patients were divided into recipients of single lung transplantation (SLT) and bilateral lung transplantation (BLT). Patients with combined heart-lung transplantation were included in the BLT group. Data for patients who underwent retransplantation were only included for the period preceding the second procedure.

\section{Statistical Analysis}

Continuous variables are reported in the text as means \pm SD. The SAS system was used for calculation. ${ }^{22}$

Changes in $F E V_{1}$ values. Statistical analysis of changes in $\mathrm{FEV}_{1}$ values (expressed as percent predicted) before and after conversion was performed by means of change-point regression analysis with a mixed linear model (SAS Proc Mixed). Slopes before and after conversion were estimated separately for recipients of SLT and BLT and were compared with the zero slope. ${ }^{22}$ The mixed-model analysis was performed separately for the RAR and stage 1 to 3 BOS (BOS1-3) groups. For the RAR group, data were fitted for the 3-month period before conversion and for the 12-month period after conversion. For the BOS groups, data were fitted for the 6-month period before and the 12-month period after conversion. All available $\mathrm{FEV}_{1}$ values within the specified periods were included in the analysis.

AR episodes. The significance of differences in the total number of AR episodes within 3 months before and 3 months after conversion were assessed by using the Wilcoxon signed-rank test. For other parameters, the Student $t$ test or Wilcoxon signed-rank test was used.

\section{Results}

\section{Patients and Immunosuppression}

Data from 280 patients were collected from 13 centers. The patients in the pre-BOS stage were excluded from the study $(\mathrm{n}=26)$. Ten patients with incomplete data or retransplantation within 2 months before conversion were excluded from the final analysis. The study thus included 244 patients, of whom $30 \%$ underwent SLT, 52\% underwent BLT, and $18 \%$ underwent heart-lung transplantation. Ten patients underwent retransplantation after the switch. Retransplantation was performed at least later than 3 months after the conversion in all 10 patients. The patients were $38 \pm 14$ years (mean $\pm \mathrm{SD}$ ) of age and included 141 male and 103 female subjects. Indications for transplantation were chronic obstructive pulmonary disease-emphysema (19\%), cystic fibrosis (29\%), primary pulmonary hypertension $(10 \%)$, idiopathic pulmonary fibrosis $(15 \%)$, and a variety of other diseases (27\%).

The RAR group included 110 patients in whom 229 episodes of rejection were diagnosed either on the basis of histology $(63 \%)$ or clinically $(37 \%)$ during the 3-month period before conversion. Within the 3-month period after conversion, 28 episodes of rejection were diagnosed either on the basis of histology (64.3\%) or clinically (35.7\%). The BOS group included 160 patients, of whom $26(16 \%)$ were in the pre-BOS stage and $134(84 \%)$ were in BOS stages 1 to 3 at the time of conversion (49 in BOS stage 1, 53 in BOS stage 2, and 32 in BOS stage 3). In the BOS1-3 group 60 patients experienced a total of 83 episodes (18\% of which were proved by means of biopsy) during the 3-month period before conversion. Within the 3-month period after conversion, a total of 17 episodes of rejection (6\% proved by means of biopsy and 94\% clinically diagnosed) were de- 
TABLE 1. Mean number of histologic and clinical episodes of acute rejection in 242 patients during a 3-month period before and after conversion from cyclosporine to tacrolimus

\begin{tabular}{|c|c|c|c|c|c|c|}
\hline & \multicolumn{3}{|c|}{ RAR } & \multicolumn{3}{|c|}{ BOS1-3 } \\
\hline & Before & After & $P$ value & Before & After & $P$ value \\
\hline Histologic rejection & $1.3 \pm 1.1(67.9 \%)$ & $0.2 \pm 0.4(64.3 \%)$ & $<.01$ & $0.1 \pm 0.4(18 \%)$ & $0.007 \pm 0.08(6 \%)$ & $<.01$ \\
\hline Clinical rejection & $0.8 \pm 1.0(32.1 \%)$ & $0.1 \pm 0.3(35.7 \%)$ & $<.01$ & $0.5 \pm 0.7(82 \%)$ & $0.1 \pm 0.3(94 \%)$ & $<.01$ \\
\hline
\end{tabular}

Data for 2 patients in the RAR group are missing. $R A R$, Recurrent-ongoing acute rejection; $B O S$, bronchiolitis obliterans syndrome.

tected in the BOS1-3 group. Median time from transplantation to conversion was 4.0 months (range, 0.5-76 months) for the RAR group and 29.3 months (range, 2.3-105 months) for the BOS group $(P<.01)$. Median observation time after conversion was 16.1 months (range, 0.7-91.3 months) for the RAR group and 18.4 months (range, 0.169.1 months) for the BOS group $(P=.58)$.

In addition to cyclosporine and steroids, 63 patients in the RAR group received azathioprine and 24 patients received mycophenolate mofetil at the time of conversion. Twenty-three patients received another immunosuppressive medication (eg, methotrexate or cyclophosphamide). In the BOS group 73 patients received azathioprine, 42 patients received mycophenolate mofetil, and 45 patients received another medication. As a whole, 168 patients in this study had received one or more courses of cytolytic therapy (antithymocyte globulin [ATG], 99\%, or antilymphocyte globulin [ALG], 1\%).

Fourteen patients in the RAR group received ATG ( $\mathrm{n}=$ 9) or OKT3 $(n=5)$ within the last 3 months before conversion as rescue therapy. In the BOS group 19 patients received cytolytic therapy (ATG, $\mathrm{n}=8$; OKT3, $\mathrm{n}=4$; RAT, $\mathrm{n}=6$; and ATG + OKT3, $\mathrm{n}=1$ ) within 3 months before conversion.

\section{Effect of Conversion in the RAR Group}

Incidence of rejection. Data on the incidence of rejection are shown in Tables 1 and 2. The incidence of histologically proved and clinically diagnosed episodes of AR decreased significantly during the 3-month period after versus before the switch from cyclosporine to tacrolimus $(P<$ $.01)$. This paralleled a reduction in the number of steroid pulses (data not shown). In 7 patients the number of episodes of AR decreased by 4 after the switch, in 18 patients it decreased by 3 , in 42 patients it decreased by 2 , and in 34 patients it decreased by 1 . In 6 patients there was no change in the number of AR episodes, and in 1 patient the number increased by 1 after the conversion.

Change in $F E V_{1}$ values. The top panel of Figure 1 shows that, on average, $\mathrm{FEV}_{1}$ values tended to decrease before conversion and to increase after conversion. Neither the preconversion slope nor the postconversion slope was significantly different from the zero slope (Figure 1, lower panel), but the change in slope between the 2 periods almost reached statistical significance $(P=.06)$. At 1 year after conversion, $67.6 \%$ of the 71 patients for whom functional data were available were still in BOS stage 0 , but $16.9 \%$ had progressed to BOS stage $1,9.9 \%$ to BOS stage 2, and $5.6 \%$ to BOS stage 3 .

\section{Effect of Conversion in the BOS1-3 Group}

Incidence of rejection. Data on the incidence of rejection are shown in Tables 1 and 2. Similar to the observation made in the RAR group, conversion to tacrolimus produced a significant reduction in the mean number of histologically proved and clinically diagnosed rejection episodes. Of the 60 patients who had AR before conversion, in 1 patient the number of episodes decreased by 4 , in 1 patient by 3 , in 12 patients by 2 , and in 37 patients by 1 when the 3-month periods before and after conversion were compared. In 5 patients there was no change in the number of AR episodes, and in 4 patients this number increased by 1 .

Change in $\mathrm{FEV}_{1}$ values. The top panel in Figure 2 shows that $\mathrm{FEV}_{1}$ values decreased substantially before conversion. Conversion from cyclosporine to tacrolimus had a dramatic effect, with a marked reduction in the rate of $\mathrm{FEV}_{1}$ decrease. On average, the rate of change in $\mathrm{FEV}_{1}$ values in the BLT recipients averaged $-3.7 \%$ of the predicted value per month before $(P<.01)$ and $-0.9 \%$ of the predicted value per month after conversion $(P<.01)$; corresponding values for SLT recipients were $-2.5 \%$ of predicted value per month $(P<.01)$ and $-0.3 \%$ of predicted value per month, respectively. The change in slope after the conversion was significant $(P<.01)$ in both BLT and SLT recipients and was observed in each of the 3 BOS groups.

\section{Infections and Renal Function}

The incidence of cytomegalovirus and noncytomegalovirus viral infections, bacterial infections, and fungal infections, as well as the total number of infectious episodes, was not significantly different for the 3-month period before and after conversion. Comparison of mean serum creatinine levels obtained during these 2 periods showed a significant increase after conversion. In the RAR group mean serum creatinine values increased from $1.3 \pm 0.9 \mathrm{mg} / 100 \mathrm{~mL}$ to $1.5 \pm 1.2 \mathrm{mg} / 100 \mathrm{~mL}(P<.01)$; corresponding values in patients converted for BOS were $1.5 \pm 0.7 \mathrm{mg} / 100 \mathrm{~mL}$ and $1.7 \pm 0.9 \mathrm{mg} / 100 \mathrm{~mL}$, respectively $(P=.04)$. 

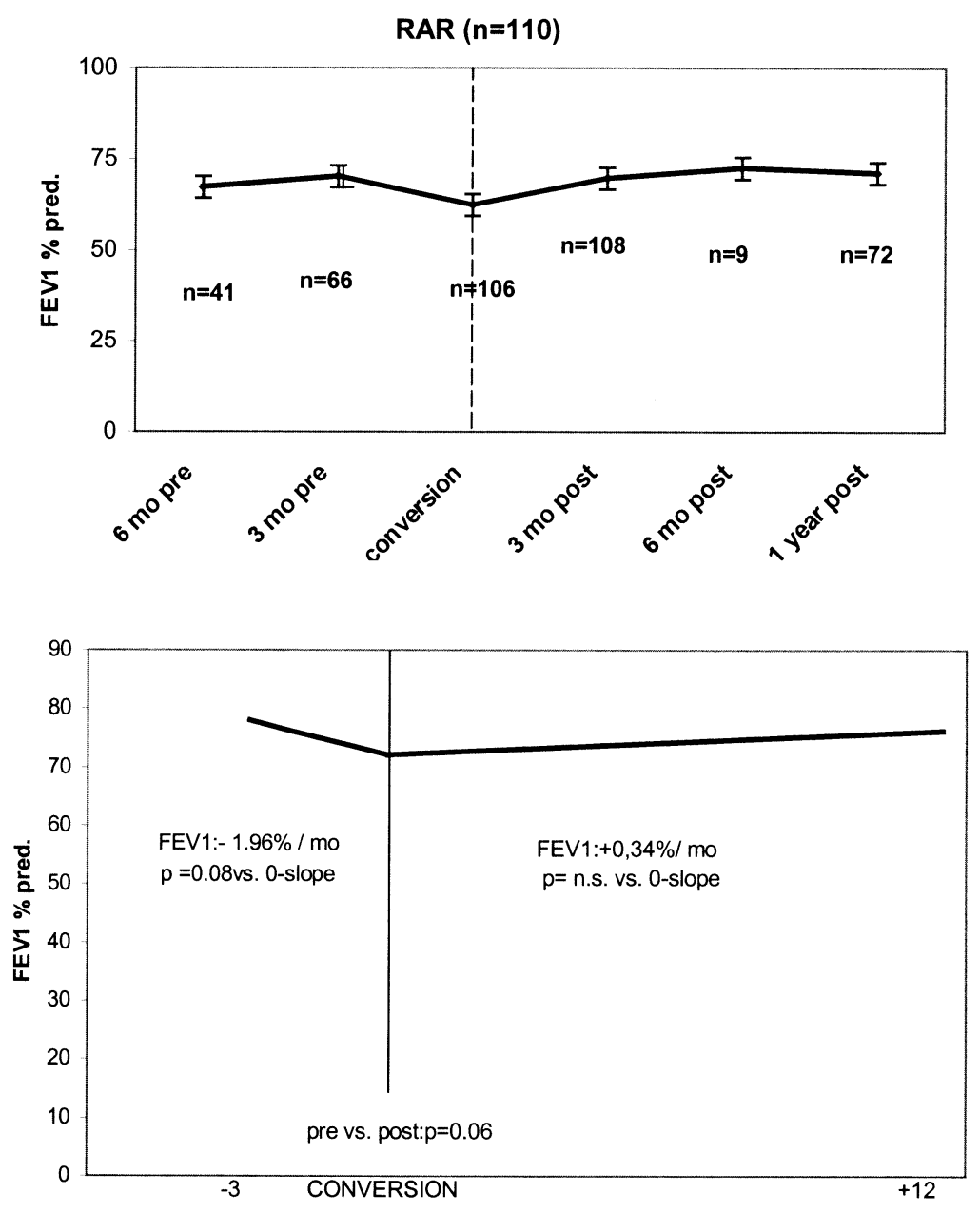

Figure 1. Upper panel, change in mean $\mathrm{FEV}_{1}$ (expressed as percent predicted) values over time in patients with RAR. Figures indicate numbers of patients for whom data were available at each time point. Bars $= \pm 1$ SEM. Lower panel, Rate of decrease of FEV, value before and after conversion.

TABLE 2. Frequency table for the differences of the total number of rejections (clinical and histologic) per patient 3 months before and 3 months after conversion from cyclosporine to tacrolimus

\begin{tabular}{lcccccr}
\hline & -4 episodes & $-\mathbf{3}$ episodes & $\mathbf{- 2}$ episodes & $\mathbf{- 1}$ episode & Unchanged & +1 episode \\
\hline RAR & 7 & 18 & 42 & 34 & 6 & 1 \\
BOS1-3 & 1 & 1 & 12 & 37 & 74 & 4 \\
\hline
\end{tabular}

$R A R$, Recurrent-ongoing acute rejection; BOS, bronchiolitis obliterans syndrome.

\section{Discussion}

Despite intense immunosuppression, acute allograft rejection is still common after lung transplantation. ${ }^{1,2}$ This susceptibility has been attributed to the fact that unlike other solid-organ transplants, the lung is constantly exposed to inhaled agents, such as antigens, toxins, irritants, and exogenous infectious agents, which promote local inflammation and might increase expression of HLA antigens. In addition, a substantial mass of lymphoid tissue containing large amounts of immune effector cells is transplanted with the graft. The Registry of the International Society for Heart and Lung Transplantation ${ }^{3}$ indicates that approximately $40 \%$ of adult and pediatric lung transplant recipients are currently treated with cyclosporine-based triple-drug immunosuppression. This regimen is effective to avoid lethal AR, but it is not powerful enough to prevent mild-to-moderate (ie, grades 2 and 3) AR. Furthermore, in a substantial proportion of patients, AR might persist or recur after an 

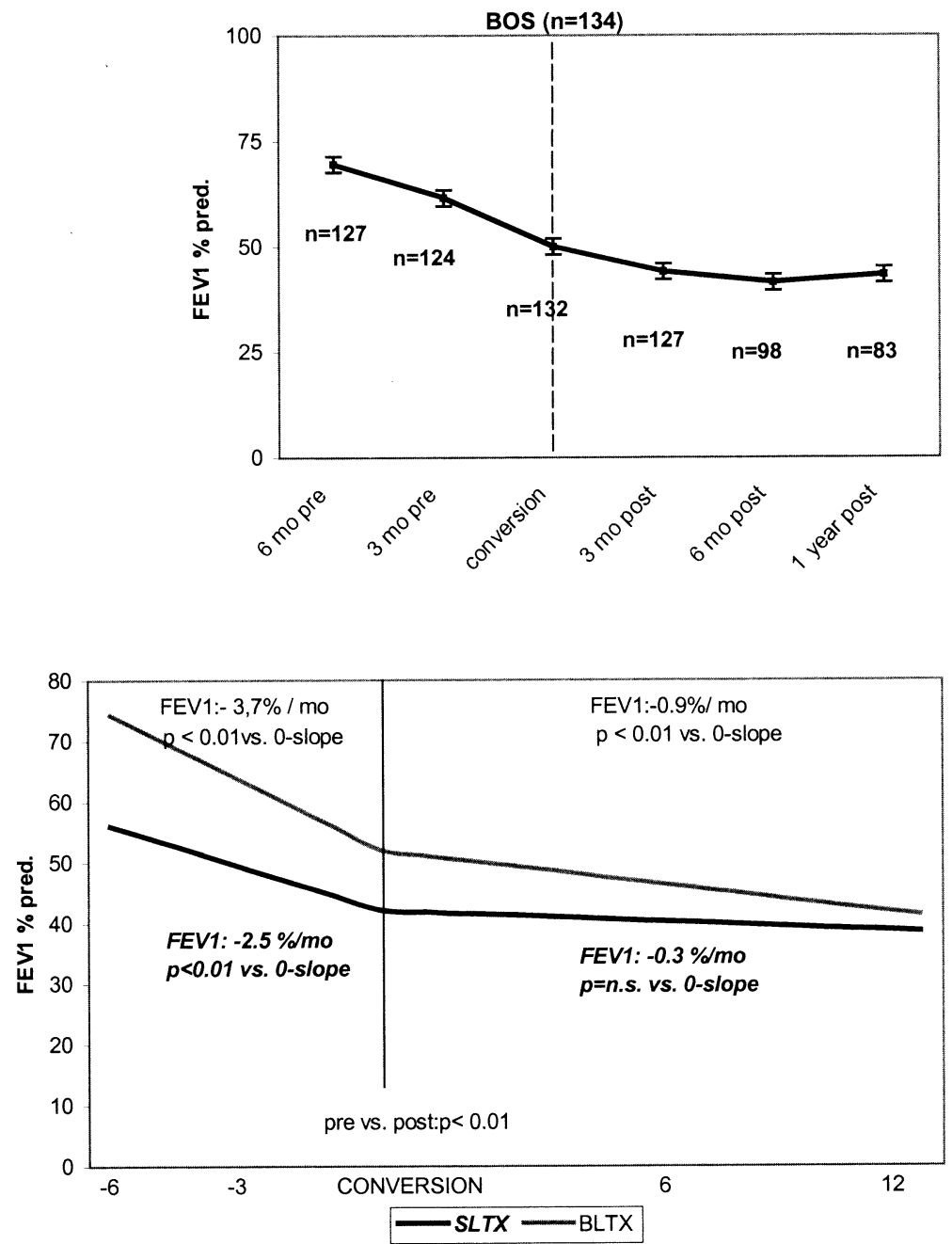

Figure 2. Data for patients in the BOS1-3 group shown in the same format as in Figure 1; the slope analysis is performed separately for SLT and BLT recipients.

initial response to treatment with high-dose methylprednisolone. In 2 previous reports ${ }^{17,23}$ more than one third of all lung transplant recipients studied had RAR. Because more frequent, long-lasting, and severe episodes of AR are the primary risk factor for BOS, ${ }^{1-8}$ it is critical to develop immunosuppressive strategies that provide a better control of RAR than cyclosporine and corticosteroids.

Two strategies have been used to control RAR. The first is to intensify immunosuppression by adding a course of cytolytic therapy, ${ }^{24}$ methotrexate, ${ }^{25}$ photopheresis ${ }^{26}$ total lymphoid irradiation, ${ }^{27}$ or aerosolized cyclosporine ${ }^{28}$ to the maintenance regimen (Table 3). The second approach is to modify the maintenance immunosuppression, for example by substituting tacrolimus for cyclosporine or mycophenolate mofetil for azathioprine. Because the available information on the efficacy of a switch from cyclosporine to tacrolimus was based on single-center reports involving small numbers of patients, we undertook this retrospective study that included data from 110 patients with RAR. Our results indicate that conversion to tacrolimus resulted in a remarkable reduction in the number of rejection episodes, irrespective of whether AR was diagnosed on the basis of histology or clinically, and in the number of pulse-steroid therapies. Overall, 94\% of patients in the RAR group experienced a decrease in the number of rejection episodes, and AR was documented in only $22 \%$ of the patients after conversion from cyclosporine to tacrolimus. In addition, conversion also markedly decreased the incidence of AR in patients with BOS.

These data confirm and extend 5 previous encouraging reports on the efficacy of tacrolimus in the treatment of RAR. ${ }^{13-17}$ Three of these reports included more than 10 patients. In the report on the randomized prospective trial of cyclosporine-based versus tacrolimus-based maintenance 
immunosuppression, Griffith and colleagues ${ }^{13}$ reported on 13 patients who were converted from cyclosporine to tacrolimus for uncontrollable multiple episodes of AR; RAR resolved in 8 patients and improved in 2 . The study from the St Louis group, ${ }^{16}$ which included 14 patients with biopsyproved AR, showed that changeover from cyclosporine to tacrolimus resulted in a marked decrease in the number of episodes of rejection per patient and per patient-day and in the average histologic grade of rejection. Similarly, the study by Onsager and associates ${ }^{17}$ from Wisconsin showed reversal of biopsy-proved AR in 8 patients and stabilization of $\mathrm{FEV}_{1}$ in 3 additional patients after conversion, whereas 4 patients did not respond to tacrolimus rescue therapy. The efficacy of tacrolimus in controlling RAR is in keeping with the observation that, when used as a primary immunosuppressive agent in solid-organ transplantation, including lung ${ }^{13,29,30}$ transplantation, tacrolimus is associated with a lower incidence of AR compared with cyclosporine.

The observation that conversion to tacrolimus provided an effective control of RAR in most patients does not imply, however, that it might prevent the later development of BOS. In the present study $32 \%$ of the 71 patients for whom functional data were available had BOS stage greater than 0 at 1 year after conversion. This was observed in only 1 of the 14 patients studied by Horning and coworkers ${ }^{16}$ but in 10 of the 15 patients studied by Onsager and associates. ${ }^{17}$ This different incidence of BOS cannot be accounted for by differences in the duration of follow-up after conversion because it was very similar in the 3 studies ( 16.1 months in the present study, 15.0 months in the study by Horning and coworkers ${ }^{16}$, and 17.1 months in the study by Onsager and associates ${ }^{17}$ ). Such a relatively short follow-up, however, does not enable one to draw any valid conclusion on the efficacy of a switch from cyclosporine to tacrolimus in the prevention of BOS.

Three previous studies have assessed the effect of conversion from cyclosporine to tacrolimus in patients with established BOS. ${ }^{18-20}$ These studies included a total of 33 patients, of whom $30 \%$ were in BOS stage $1,30 \%$ were in BOS stage 2, and $40 \%$ were in BOS stage 3 . In each study conversion from cyclosporine to tacrolimus significantly decreased the monthly rate of decrease in $\mathrm{FEV}_{1}$, which ranged from -69 to $-160 \mathrm{~mL} / \mathrm{mo}$ before the switch and from -24 to $-30 \mathrm{~mL} / \mathrm{mo}$ after the switch. The present data show that these gratifying results are confirmed when a much larger group of patients is studied. The beneficial effect of the conversion on lung function was observed in each BOS group, with stabilization of $\mathrm{FEV}_{1}$ values, and in both SLT and BLT recipients (Figure 2).

As in previous studies, ${ }^{16,17}$ no major adverse effects of tacrolimus treatment were encountered. The incidence of infections did not increase after conversion. The slight increase in serum creatinine values in the 3 -month period after
TABLE 3. Different strategies to treat recurrent-ongoing acute rejection

\begin{tabular}{rlr}
\hline $\begin{array}{l}\text { No. of } \\
\text { treated } \\
\text { patients }\end{array}$ & \multicolumn{1}{c}{ Therapy } & $\begin{array}{r}\text { Rate of } \\
\text { success }\end{array}$ \\
\hline 1 & OKT3 & $100 \%$ \\
6 & Total Iymphoid irradiation & $21 \%$ \\
9 & Aerosolized cyclosporine & $78 \%$ \\
12 & Methotrexate & $83 \%$ \\
14 & Photopheresis & $21 \%$ \\
\hline
\end{tabular}

conversion might be related to the nephrotoxicity of tacrolimus, in particular when trough levels are in the upper portion of the therapeutic range. Alternatively, it might reflect progression of an already established impairment of kidney function because of the previous use of cyclosporine.

The present study is limited by its retrospective design, the number of missing data, and the absence of a control group. Because the risk of rejection decreases with time after the operation, we cannot exclude that the reduced incidence of AR after conversion to tacrolimus was coincidental.

We also cannot exclude that the beneficial effect that is attributed to the conversion from cyclosporine to tacrolimus might represent the collective effect of the change in calcineurin inhibitor plus other contemporaneous interventions.

Similarly, because the rate of loss of lung function in many obstructive lung diseases is nonlinear, with the rate of decrease in $\mathrm{FEV}_{1}$ decreasing as airflow obstruction becomes more severe, the possibility exists that the stabilization of lung function observed in patients with BOS after conversion might have been observed without changing medications. However, the fact that the beneficial effects of conversion were robustly observed in a large group of patients makes a cause-effect relationship plausible. Thus the present findings should be interpreted as suggestive that conversion from cyclosporine to tacrolimus is associated with reversal of RAR and with slowing of functional loss in most patients with established BOS. These results should now be further corroborated by a prospective randomized trial in a large number of lung transplant recipients before advocacy of widespread tacrolimus prescription for control of RAR and BOS.

We acknowledge the contributions from S. Keeshavjee, C. Aboyoun, Greg I. Snell, J. Wardle, and H. Treede. We thank Fujisawa Company for supporting the collection of data.

\section{References}

1. Paradis I. Bronchiolitis obliterans: pathogenesis, prevention, and management. Am J Med Sci. 1998;315:161-78. 
2. Boehler A, Estenne M. Obliterative bronchiolitis after lung transplantation. Curr Opin Pulm Med. 2000;6:133-9.

3. Hertz MI, Taylor DO, Trulock EP, et al. The registry of the international society for heart and lung transplantation: nineteenth official report-2002. J Heart Lung Transplant. 2002;21:950-70.

4. Reichenspurner H, Girgis RE, Robbins RC, et al. Stanford experience with obliterative bronchiolitis after lung and heart-lung transplantation. Ann Thorac Surg. 1996;62:1467-72.

5. Whitehead B, Rees P, Sorensen K, et al. Incidence of obliterative bronchiolitis after heart-lung transplantation in children. J Heart Lung Transplant. 1993;12:903-8.

6. Girgis RE, Tu I, Berry GJ, et al. Risk factors for the development of obliterative bronchiolitis after lung transplantation. J Heart Lung Transplant. 1996;15:1200-8.

7. Sharples LD, Tamm M, McNeil K, Higenbottam TW, Stewart S, Wallwork J. Development of bronchiolitis obliterans syndrome in recipients of heart-lung transplantation-early risk factors. Transplantation. 1996;61:560-6.

8. Bando K, Paradis IL, Similo S, et al. Obliterative bronchiolitis after lung and heart-lung transplantation. J Thorac Cardiovasc Surg. 1995; 110:4-14.

9. Gote T, Kino T, Hatanaka H, et al. FK506: Historical perspectives. Transplant Proc. 1991;23:271-3.

10. Woodle ES, Thistlewaite JR, Gordon JH, et al. A multicenter trial of FK506 (tacrolimus) therapy in refractory acute renal allograft rejection: a report of the tacrolimus kidney transplantation rescue study group. Transplantation. 1996;62:594-9.

11. McDiarmid SV, Klintmalm GB, Busuttil RW. FK506 conversion for intractable rejection of liver allograft. Transplant Int. 1993;6:305-12.

12. Armitage JM, Kormos RL, Fung J, Starzl TE. Clinical trial of FK506 as primary and rescue immunosuppression in adult cardiac lung transplantation. Transplant Proc. 1991;23:3054-7.

13. Griffith BP, Bando K, Hardesty RL, et al. A prospective randomised trial of FK 506 versus cyclosporine after human pulmonary transplantation. Transplantation. 1994;57:848-51.

14. Knoop C, Antoine M, Vachiery JL, Yernault JC, Estenne M. FK506 rescue therapy for irreversible airway rejection in heart-lung transplant recipients: report on five cases. Transplant Proc. 1994;26:3240-1.

15. Mentzer RM, Jahania MS, Lasley RD, US Multicenter FK506 Study Group. Tacrolimus as a rescue immunosuppressant after heart and lung transplantation. Transplantation. 1998;65:109-13.

16. Horning NR, Lynch JP, Sundaresan SR, Patterson GA, Trulock EP. Tacrolimus therapy for persistent or recurrent acute rejection after lung transplantation. J Heart Lung Transplant. 1998;17:761-7.

17. Onsager DR, Canver CC, Jahania MS, et al. Efficacy of tacrolimus in the treatment of refractory rejection in heart and lung transplant recipients. J Heart Lung Transplant. 1999;18:448-55.

18. Kesten S, Chaparro C, Scavuzzo M, Gutierrez C. Tacrolimus as rescue therapy for bronchiolitis obliterans syndrome. J Heart Lung Transplant. 1997;16:905-12.

19. Ross DJ, Lewis MI, Kramer M, et al. FK506 rescue immunosuppression for obliterative bronchiolitis after lung transplantation. Chest. 1997;112:1175-9.

20. Revell MP, Lewis ME, Llewellyn-Jones CG, Wilson IC, Bonser RS. Conservation of small-airway function by tacrolimus/cyclosporine conversion in the management of bronchiolitis obliterans following lung transplantation. J Heart Lung Transplant. 2000;19:1219-23.

21. Cooper J, Billingham M, Egan T, et al. A working formulation for the standardization of nomenclature and for clinical staging of chronic dysfunction in lung allografts. J Heart Lung Transplant. 1993;12: 713-6.

22. SAS Institute Inc. SAS/STAT user's guide, version 8. Cary, NC: SAS Institute Inc; 1999. p. 3884.

23. Guilinger RA, Paradis IL, Dauber JH, et al. The importance of bronchoscopy with transbronchial lung biopsy and bronchoalveolar lavage in the management of lung transplant recipients. Am J Respir Crit Care Med. 1995;152:2037-43.

24. Shennib H, Mercado M, Nguyen D, et al. Successful treatment of steroid-resistant double-lung allograft rejection with orthoclone OKT3. Am Rev Respir Dis. 1991;141:224-6.

25. Cahill BC, O'Rourke MK, Strasburg KA, et al. Methotrexate for lung transplant recipients with steroid-resistant acute rejection. J Heart Lung Transplant. 1996;15:1130-7.

26. Villanueva J, Bhorade SM, Robinson JA, Husain AN, Garrity ER Jr. Extracorporal photopheresis for the treatment of lung allograft rejection. Ann Transplant. 2000;5:44-7.

27. Valentine VG, Robbins RC, Wehner JH, Patel HR, Berry GJ, Theodore J. Total lymphoid irradiation for refractory acute rejection in heart-lung transplantation. Chest. 1996;109:1184-9.

28. Iacono AT, Keenan RJ, Duncan SR, et al. Aerosolized cyclosporine in lung recipients with refractory rejection. Am J Respir Crit Care Med. 1996;153:1451-5.

29. Keenan RJ, Konishi H, Kawaki A, et al. Clinical trial of tacrolimus versus cyclosporin in lung transplantation. Ann Thorac Surg. 1995;60: 580-5.

30. Zuckermann A, Reichenspurner H, Klepetko W, et al. CyclosporineA (CsA) versus Tacrolimus (Tac), in combination with mycophenolate mofetil (MMF) and steroids as primary immunosuppression after lung transplantation: one-year results of a 2-center prospective randomised trial. J Thorac Cardiovasc Surg. 2003;125(4):891-900. 